
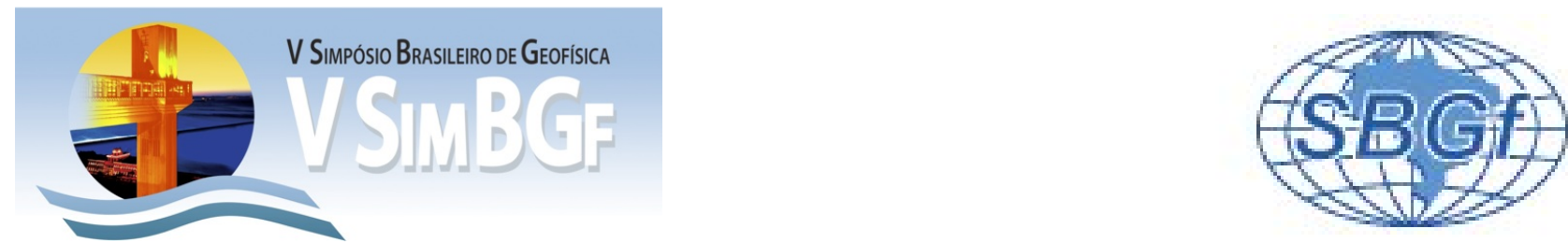

\title{
Atenuação do ground roll utilizando a transformada wavelet em conjunto com a de- convolução adaptativa
}

Lucas J. A. de Almeida* e Milton J. Porsani, CPGG-UFBA/INCT-GP/CNPq

Copyright 2012, SBGf - Sociedade Brasileira de Geofísica Este texto foi preparado para a apresentação no $V$ Simpósio Brasileiro de Geofísica, Salvador, 27 a 29 de novembro de 2012. Seu conteúdo foi revisado pelo Comitê Técnico do $V$ SimBGf, mas não necessariamente representa a opinião da SBGf ou de seus associados. É proibida a reprodução total ou parcial deste material para propósitos comerciais sem prévia autorização da SBGf.

\section{RESUMO}

Neste trabalho, apresentamos um método de atenuação do ground roll que utiliza a transformada wavelet em conjunto com a deconvolução adaptativa. A transformada wavelet é utilizada de modo a dividir o sismograma em bandas de coeficientes de tipos diferentes, com o objetivo de localizar melhor o ground roll, que é um ruído de baixa frequência e alto mergulho.

Em conjunto com a transformada wavelet, a deconvolução adaptativa é aplicada na banda que contém o ground roll, de modo a eliminar somente o ruído coerente e aumentar a razão sinal/ruído. Ilustramos o método aplicando-o nos dados do tiro 39 que compõe uma linha sísmica terrestre da bacia de Tacutu. Os resultados mostram que o método funciona bem e permite colocar em evidência reflexões que antes estavam mascaradas pelo ground roll.

\section{INTRODUÇÃO}

O ground roll é um tipo de onda de superfície que possui alta amplitude e baixa frequência, e está comumente presente como um ruído coerente em dados sísmicos terrestres, ocultando as reflexões de interesse no sismograma. Vários métodos já foram desenvolvidos na tentativa de atenuar este tipo de ruído. Um dos métodos clássicos é a filtragem $f-k$, que usa a transformada de Fourier 2D para passar o dado para o domínio frequência-número de onda. Como, neste domínio, o ground roll é representado em linhas, este pode ser removido por meio da filtragem passa banda.

Recentemente, o tratamento do ground roll utilizando a transformada wavelet, tanto em 1D (Deighan e Watts, 1997) como em 2D (Miao e Cheadle, 1998), e (Matos e Osorio et al., 2002), foi objeto de pesquisa de diver- sos autores. Em alguns deles, o procedimento adotado é zerar alguns coeficientes da banda na qual o ground roll se encontra, enquanto outros propõem algum tipo de filtragem associada à transformada wavelet para a atenuação do mesmo, como o filtro hiperbólico de velocidade (Miao e Cheadle, 1998).

No presente trabalho, apresentamos um método de filtragem do ground roll baseado na transformada wavelet em conjunto com a deconvolução adaptativa. Aplicamos o método, para fins ilustrativos, no tiro 39 de uma linha sísmica terrestre da bacia de Tacutu.

\section{A TRANSFORMADA WAVELET DISCRETA 2D}

Utilizamos, neste trabalho, o método não-convencional de decomposição da transformada wavelet discreta 2D (Matos e Osorio et al., 2002), conforme mostrado na figura 1 (Wu e Chen et al., 2001). Com este método, procuramos fazer a análise do sismograma de acordo com a teoria da multiresolução (Mallat, 1989), fazendo a decomposição do sismograma original utilizando um par de filtros, um passa-baixa e um passa-alta, que representam a wavelet escolhida para fazer a transformação. O filtro passa-baixa atua fazendo a aproximação na dimensão na qual ele é utilizado, enquanto o filtro passa-alta atua fazendo a diferenciação. Os filtros associados à wavelet Haar, que foi a wavelet utilizada neste trabalho, estão denotados no conjunto de equações (1), onde $\mathbf{G}$ representa os coeficientes do filtro passa-baixa, e $\mathbf{H}$ representa os coeficientes do filtro passa-alta ( $\mathrm{Vi}$ dakovic e Muller et al., 1994).

$$
\begin{aligned}
\mathbf{G}(0) & =\frac{1}{\sqrt{2}} \\
\mathbf{G}(1) & =\frac{1}{\sqrt{2}} \\
\mathbf{H}(0) & =\frac{1}{\sqrt{2}} \\
\mathbf{H}(1) & =\frac{-1}{\sqrt{2}}
\end{aligned}
$$

O resultado da decomposição pode ser observado esquematicamente na figura 2. As bandas decompostas estão representadas pelas letras $L$ e $H$, onde $L$ faz menção à aplicação do filtro passa-baixa e $\mathrm{H}$, ao filtro passa-alta; 


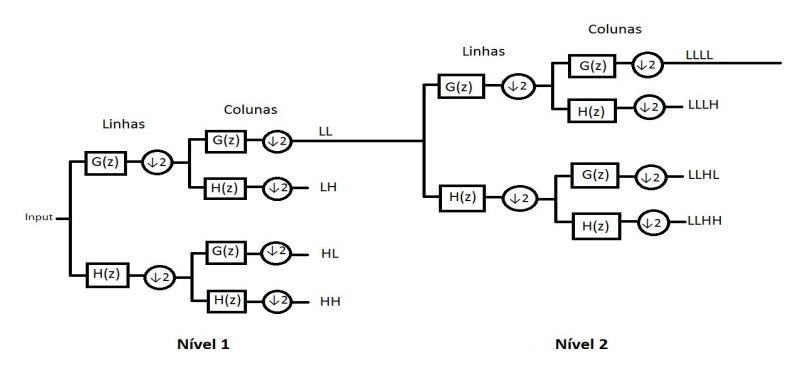

Figura 1: Decomposição segundo o método nãoconvencional com a transformada discreta wavelet 2D. $\mathbf{G}$ representa o filtro passa-baixa, e $\mathbf{H}$ representa o filtro passa-alta.

na ordem a qual os filtros foram utilizados em cada uma. As bandas com somente duas letras pertencem ao primeiro nível de decomposição, as com quatro letras pertencem ao segundo nível, e assim por diante; cada uma com metade do número de amostras do nível anterior.

Como as bandas de final HL foram geradas pela aproximação do sinal em $t$ e sua diferenciação em $x$, estas são denominadas de bandas de coeficientes verticais. Do mesmo modo, as bandas de final LH são chamadas de bandas de coeficientes horizontais, e as de final $\mathrm{HH}$, de bandas de coeficientes diagonais (Matos e Osorio et al., 2002). Como o ground roll se manifesta na forma de um cone vertical de alta amplitude e baixa frequência, este, na decomposição utilizando a transformada wavelet, aparecerá mais fortemente nas bandas de coeficientes verticais, ou de final HL.

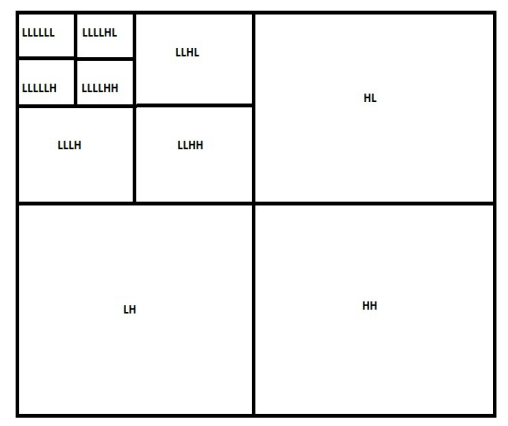

Figura 2: Representação das bandas de coeficientes em uma decomposição de três níveis.

\section{DECONVOLUÇÃO ADAPTATIVA}

A deconvolução adaptativa, neste trabalho, é utilizada de modo a filtrar o ruído, traço a traço, do sismograma original. A aplicação se dá através de uma janela móvel, que contém $\mathrm{L}$ coeficientes e se desloca de um coeficiente no traço para cada iteração, como é mostrado na figura 3. Para cada janela de $L$ coeficientes, o sistema de equações mostrado na equação (2) é resolvido, e os filtros causal $\left(a_{t}\right)$ e anti-causal $\left(b_{t}\right)$ são definidos, de modo que o erro seja mínimo.

O sistema disposto na equação (2) considera filtros $a_{t}$ e $b_{t}$ com 2 coeficientes, embora seja possível ser utilizado tantos quanto necessários, fazendo as devidas modificações. Para esta aplicação, o sistema de equações considerado difere do sistema de equações proposto pela deconvolução clássica de Wiener-Levinson, pois não faz suposições acerca das amplitudes nos tempos fora da janela considerada, ou seja, não contém zeros fora da janela. Isto faz com que os filtros causal e anti-causal não sejam iguais (Porsani et al., 1992).

$$
\begin{aligned}
{\left[\begin{array}{cc}
\mathbf{e}_{a, 3} & \mathbf{e}_{b, 1} \\
\cdot & \cdot \\
\cdot & \cdot \\
\mathbf{e}_{a, t} & \mathbf{e}_{b, t-2} \\
\cdot & \cdot \\
\cdot & \cdot \\
\mathbf{e}_{a, l} & \mathbf{e}_{b, l-2}
\end{array}\right] } & =\left[\begin{array}{ccc}
x_{3} & x_{2} & x_{1} \\
\cdot & \cdot & \cdot \\
\cdot & \cdot & \cdot \\
x_{t} & x_{t-1} & x_{t-2} \\
\cdot & \cdot & \cdot \\
\cdot & \cdot & \cdot \\
x_{l} & x_{l-1} & x_{l-2}
\end{array}\right] \\
* & {\left[\begin{array}{cc}
1 & b_{2} \\
a_{1} & b_{1} \\
a_{2} & 1
\end{array}\right] }
\end{aligned}
$$

Após a definição dos filtros causal e anti-causal, definimos $\mathbf{e}_{t}$, ou erro absoluto, para um dado tempo $t$, como a diferença entre os erros do filtro causal e anti-causal, como está descrito no conjunto de equações (3).

$$
\begin{aligned}
\mathbf{e}_{t} & =\mathbf{e}_{a, t}-\mathbf{e}_{b, t} \\
\mathbf{e}_{a, t}-\mathbf{e}_{b, t} & =a_{1} x_{t-1}+a_{2} x_{t-2}-b_{1} x_{t+1}-b_{2} x_{t+2}
\end{aligned}
$$

Da equação (3), podemos observar que o filtro que será utilizado na filtragem dos dados, denotado como $\mathbf{h}_{t}$, que é um filtro não causal, é formulado como está escrito na equação (4).

$$
\mathbf{h}_{t}=\left\{-b_{2},-b_{1}, 0, a_{1}, a_{2}\right\}
$$

A filtragem, então, é feita traço a traço de acordo com a figura 3 , utilizando o filtro $\mathbf{h}_{t}$, que é único para cada janela de $L$ coeficientes, em cada janela móvel. Cada janela de $L$ coeficientes do traço original fornece $L$ amostras do traço filtrado, e cada amostra do traço filtrado, em um determinado tempo t, é obtida com o valor médio da filtragem das amostras de cada janela, neste mesmo tempo. 


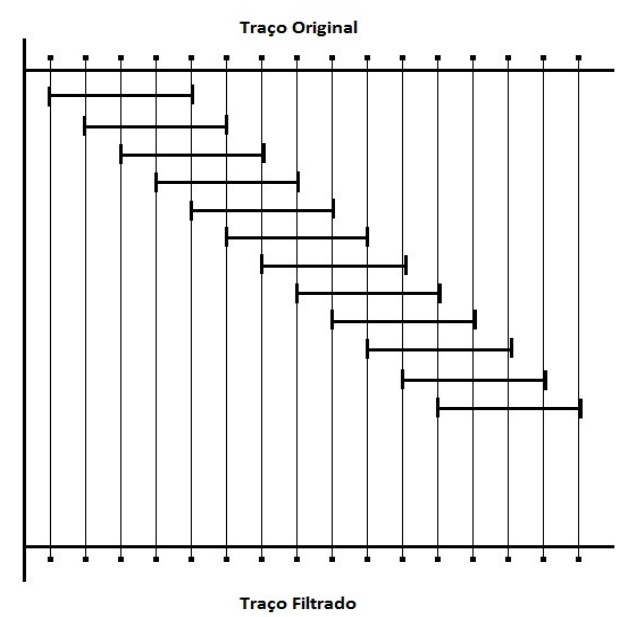

Figura 3: Representação da deconvolução adaptativa. Para cada tempo, a amostra filtrada final é a média das amostras filtradas, naquele tempo, em todas as janelas móveis as quais a linha vertical que liga a amostra original com a amostra filtrada final intercepta.

\section{METODOLOGIA}

Com base nas seções acima, podemos então descrever o seguinte processo para empregar as técnicas mencionadas anteriormente:

- Decomposição do sismograma utilizando a transformada wavelet em quantos níveis forem necessários;

- Reconhecimento das bandas de coeficientes verticais;

- Aplicação da deconvolução adaptativa utilizando uma janela e um filtro do tamanho desejado, somente nas bandas de coeficientes verticais;

- Reconstrução do sismograma utilizando a transformada wavelet inversa.

\section{RESULTADOS}

Aplicamos o método abordado anteriormente ao tiro 39, proveniente da bacia de Tacutu. De modo a filtrar o ground roll, o tiro foi decomposto utilizando a transformada wavelet em três níveis utilizando a wavelet Haar. A seguir, isolamos as bandas de coeficientes verticais de cada nível de decomposição, e aplicamos a deconvolução adaptativa utilizando um filtro de 2 coeficientes e uma janela móvel de 20 amostras nas mesmas. A figura 4 mostra o tiro 39 original, e o mesmo tiro após a filtragem proposta no trabalho. Como forma de comparação, a figura 5 mostra o mesmo sismograma filtrado diretamente com a deconvolução adaptativa, sem a utilização da transformada wavelet; e o sismograma filtrado utilizando a transformada wavelet em conjunto com o mute da banda de coeficientes verticais, na qual o ground roll se manifesta mais fortemente.

Como se pode notar, a filtragem somente com a utilização da deconvolução adaptativa se mostrou inferior, já que o ground roll foi atenuado, mas as reflexões que estavam ocultas por ele não apareceram. Quanto ao sismograma que utiliza o método de mute da banda associada ao ground roll, podemos ver que este ficou bastante parecido com o sismograma proveniente da filtragem proposta no trabalho; embora os eventos da parte superior do sismograma, como as ondas diretas, tenham sido mais afetados pelo processo.

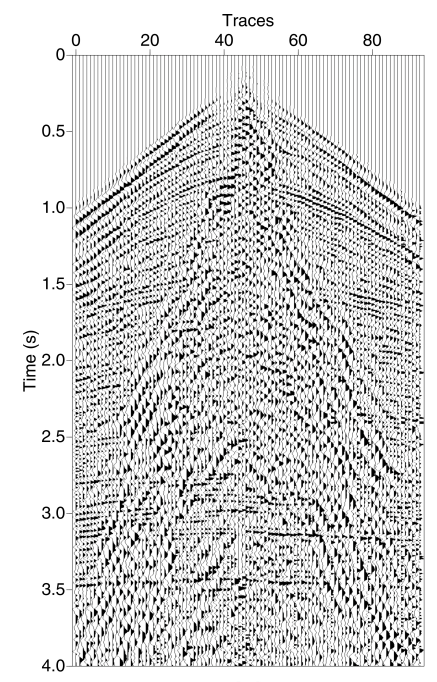

(a)

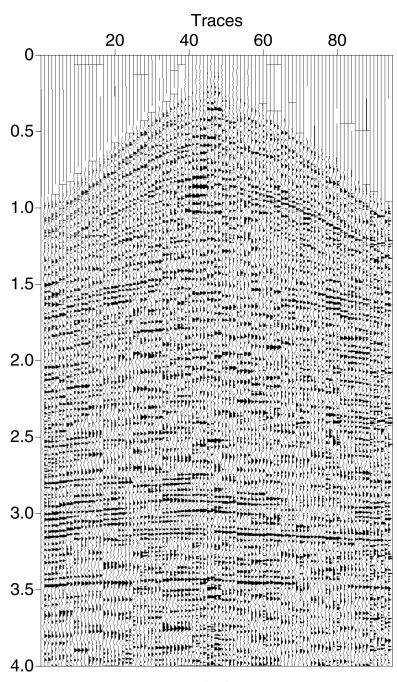

(b)
Figura 4: (a) - Sismograma do tiro 39 original. (b) - Sismograma após a filtragem com a transformada wavelet em conjunto com a deconvolução adaptativa.

\section{DISCUSSÃO E CONCLUSÕES}

A transformada wavelet discreta 2D, em conjunto com a deconvolução adaptativa, mostrou ser um método bastante eficiente na atenuação do ground roll. Embora, no presente trabalho, seja feito o uso da wavelet Haar; os resultados foram satisfatórios quando comparados com alguns dos métodos de filtragens clássicos já existentes, como a filtragem $f-k$, ou mesmo a deconvolução sem o apoio da transformada wavelet. Entretanto, a utilização de uma wavelet de fase linear e com maior suavidade pode trazer melhoras significativas para o método.

\section{AGRADECIMENTOS}

Os autores agradecem ao INCT-GP/CNPq e Lucas J. A. de Almeida ao CPGG/IGEO/UFBA, pelas facilidades 


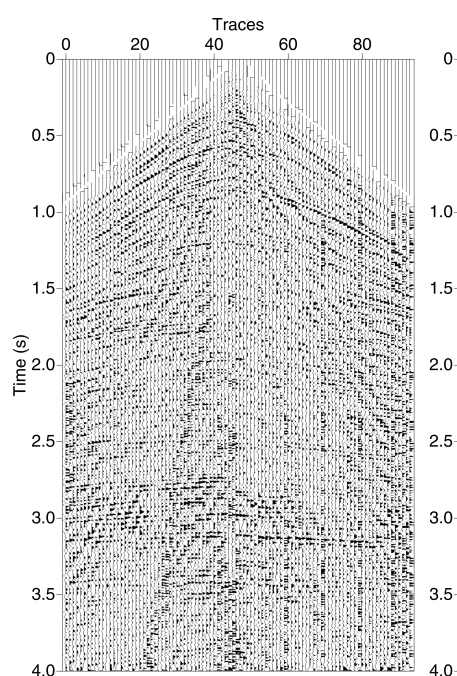

(a)

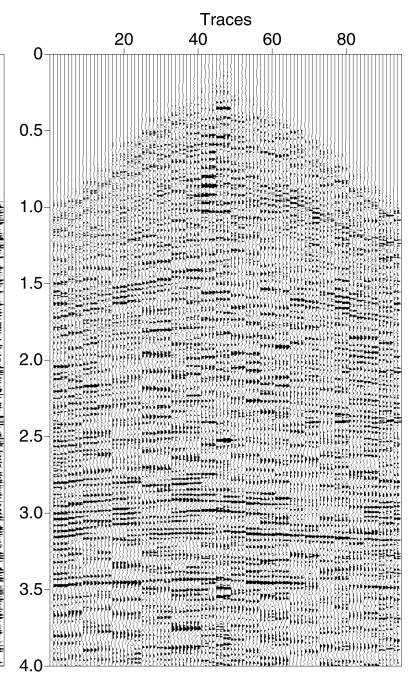

(b)

Figura 5: (a) - Sismograma do tiro 39 após a filtragem somente com a deconvolução adaptativa. (b) Sismograma após a filtragem com o mute das bandas de coeficientes verticais.

oferecidas para o desenvolvimento do trabalho.

\section{REFERÊNCIAS}

Deighan, A. J., e Watts, D. R., 1997, Ground-roll supression using the wavelet transform: Geophysics, 62, 1896-1903.

Mallat, S., 1989, A theory for multiresolution signal decomposition: The wavelet representation: Trans. on Pattern Analysis and Machine Intelligence, IEEE, 7, 674-693.

Matos, M. C., e Osorio, P. L. M., 2002, Wavelet transform filtering in the $1 \mathrm{~d}$ and $2 \mathrm{~d}$ for ground roll suppression: SEG Annual Meeting.

Miao, X., e Cheadle, S., 1998, Noise attenuation with wavelet transforms: SEG Annual Meeting.

Porsani, M. J., 1992, Effective solution of covariance equations with applications to seismic trace extrapolation and predictive deconvolution: SEG Annual Meeting.

Vidakovic, B., e Muller, P., 1994, Wavelets for kids: unpublished.

Wu, P. C., e Chen, L. G., 2001, An efficient architecture for two-dimensional discrete wavelet transform: Circuits and Systems for Video Technology, IEEE. 